\title{
A Tunable Wideband Frequency Synthesizer Using LC-VCO and Mixer for Reconfigurable Radio Transceivers
}

\author{
Yusaku Ito, ${ }^{1}$ Kenichi Okada, ${ }^{2}$ and Kazuya Masu ${ }^{1}$ \\ ${ }^{1}$ ICE Cube Center, Tokyo Institute of Technology, Tokyo 226-8503, Japan \\ ${ }^{2}$ Department of Physical Electronics, Tokyo Institute of Technology, Tokyo 152-8552, Japan
}

Correspondence should be addressed to Kenichi Okada, okada@ssc.pe.titech.ac.jp

Received 2 May 2011; Accepted 6 June 2011

Academic Editor: Antonio Liscidini

Copyright ( 2011 Yusaku Ito et al. This is an open access article distributed under the Creative Commons Attribution License, which permits unrestricted use, distribution, and reproduction in any medium, provided the original work is properly cited.

\begin{abstract}
This paper proposes a novel wideband LC-based voltage-controlled oscillator (VCO) for multistandard transceivers. The proposed VCO has a core LC-VCO and a tuning-range extension circuit, which consists of switches, a mixer, dividers, and variable gain combiners with a spurious rejection technique. The experimental results exhibit 0.98 to $6.6 \mathrm{GHz}$ continuous frequency tuning with $-206 \mathrm{dBc} / \mathrm{Hz}$ of $\mathrm{FoM}_{\mathrm{T}}$, which is fabricated by using a $0.18 \mu \mathrm{m}$ CMOS process. The frequency tuning range (FTR) is $149 \%$, and the chip area is $800 \mu \mathrm{m} \times 540 \mu \mathrm{m}$.
\end{abstract}

\section{Introduction}

Recently, dozens of wireless communication standards have been used for small mobile terminals, for example, GSM, UMTS, LTE, WiMAX, WLAN, Bluetooth, UWB, GPS, DTV, and RFID, and the standards use several frequency bands spreading in a quite wide range such as $800 \mathrm{MHz}$ to $6 \mathrm{GHz}$. The mobile terminals have been obtaining multistandard operations, smaller size, and lower power operation [12]. However, the present multistandard RF front end consists of several LNAs, VCOs, mixers, and PAs for each frequency band (Figure 1). A multistandard RF front end implemented in a single chip is required for smaller size, lower power, and more flexible wireless communication terminals such as $800 \mathrm{MHz}$ to $6 \mathrm{GHz}$. The software defined radio (SDR) has been studied $[9,13]$, and the multistandard RF front end is also needed to realize the SDR with feasible power consumption. Several multistandard RF front ends have been proposed. Digital-assist architectures are suitable for Si CMOS chips $[14,15]$. As a common component for the multistandard RF front ends, this paper proposes a wideband frequency synthesizer covering $0.98 \mathrm{GHz}$ to $6.6 \mathrm{GHz}$ [20].

\section{Previous Work}

Ring-oscillator-based VCOs have unacceptably large phase noise for the wireless communication while it has very wide frequency tuning range. Thus, LC-based VCOs are required for the application due to the phase noise requirement. However, the tuning range of LC-based VCOs is usually very narrow such as 2 to $3 \mathrm{GHz}$ even through the $800 \mathrm{MHz}$-to$6 \mathrm{GHz}$ tuning range is required for the multistandard $\mathrm{RF}$ front ends. The conventional LC-VCO cannot overcome the trade-off, so a new wideband LC-based VCO architecture has to be developed.

A VCO using switched capacitors is a well-known topology to extend the tuning range $[7,21]$, and a switched inductor and a variable active inductor are also utilized $[8,16]$. However, these circuits have a trade-off between the phase noise and the tuning range. The VCO using a variable MEMS inductor achieves wide-tuning range with superior phase noise characteristics [18]. However, it is difficult for these pure CMOS VCOs to obtain wide-tuning range with adequate phase noise.

Recently, wideband VCOs for MB-OFDM UWB have been reported $[1,2,4,17,22,26]$, which use a tuning range extension technique using QVCO, dividers, and singlesideband mixer (SSBM). These VCOs achieve quite wide tuning range and high spurious rejection using SSBM with $I / Q$ signals. However, the VCOs in $[1,2]$ use two oscillators and have large layout area and larger power consumption. Although the VCOs in $[10,22,26]$ use only one QVCO, these VCOs also have larger phase noise and larger power consumption. 


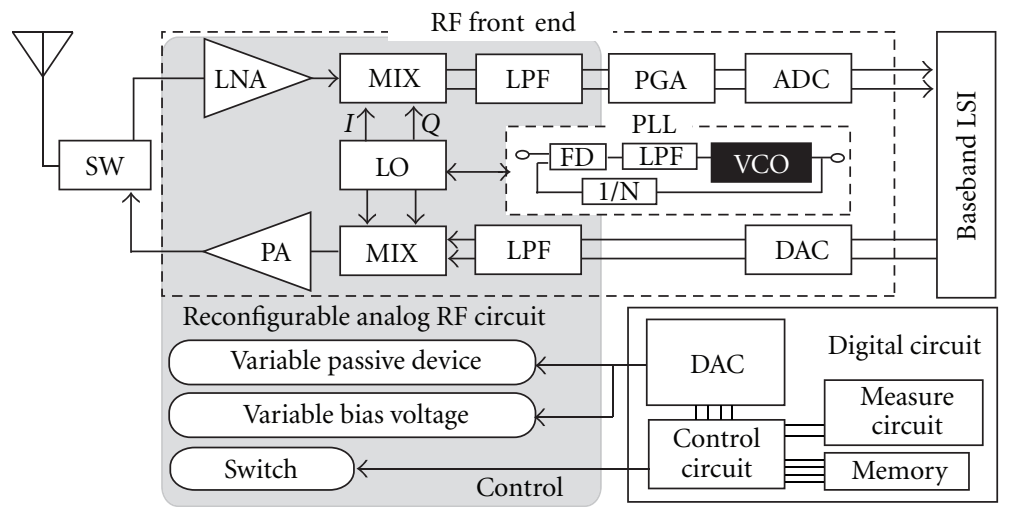

FIgURE 1: Concept of the reconfigurable RF circuit design.

If switch turns to (B), gain $\mathrm{B}$ is controlled to zero
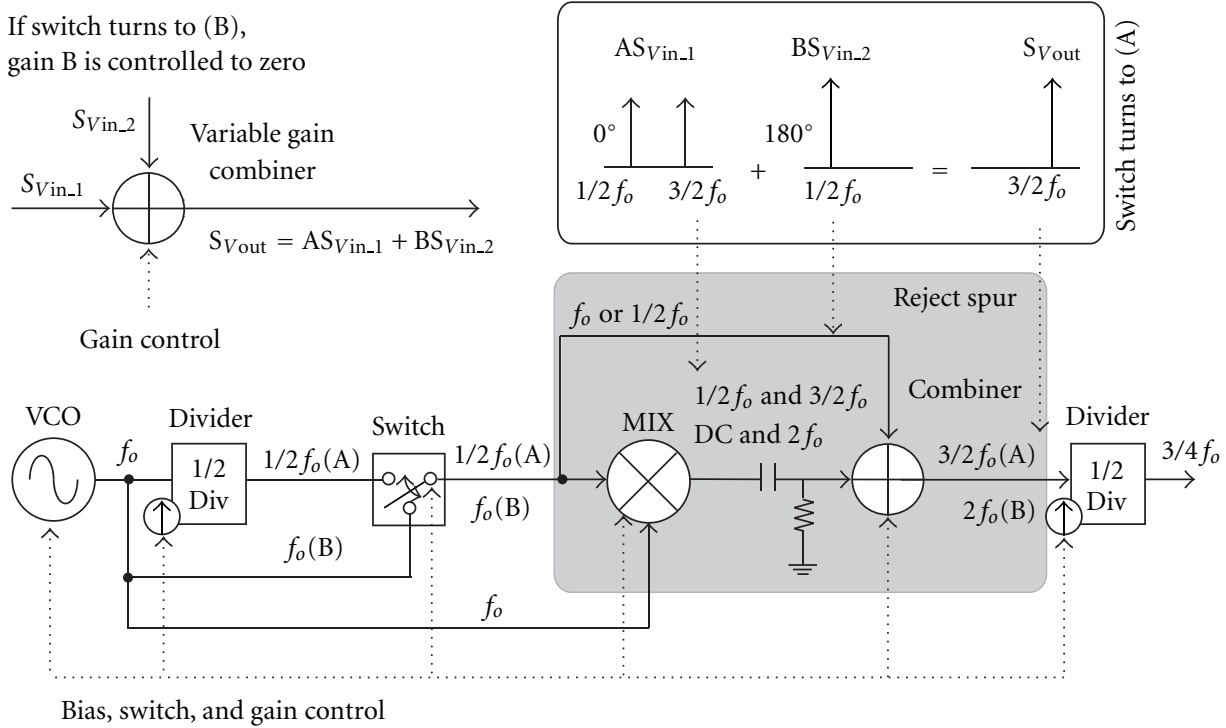

FIGURE 2: The proposed wideband VCO architecture.

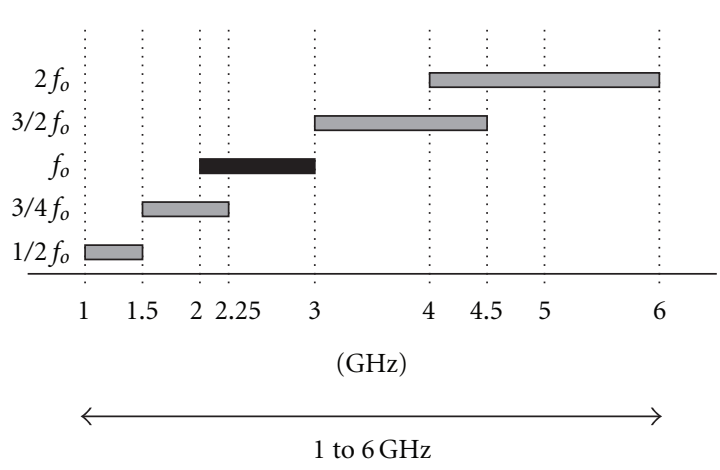

Figure 3: Frequency plan from $1 \mathrm{GHz}$ to $6 \mathrm{GHz}$.

Wideband VCOs for multistandard transceivers are also reported $[10,13,23]$. The VCO in [10] use a QVCO and SSBMs, which also has larger phase noise and larger power consumption. The VCOs in $[13,23]$ use differential oscillators and $1 / 2$ frequency dividers to avoid utilizing SSBM and the quadrature generation. The VCO in [13] uses two oscillators, and it requires, moreover, three oscillators for continuous frequency tuning. The VCO in [23] still requires two oscillators.

The wideband VCO proposed in [19] uses divide-by2 , divide-by-3, divide-by-4, divide-by-5, divide-by-6, divideby- 8 , and divide-by-10 frequency dividers for the tuning range extension. This architecture requires a wideband QVCO, and continuous tuning cannot be realized in the measurement [19] because $\pm 20 \%$ tuning range is difficult for QVCOs.

Various topologies for tuning range extension can be utilized depending on the required performances. In this paper, we propose a novel extension architecture to achieve wider tuning range with lower power, smaller layout area, and lower phase noise, which achieves $\pm 71 \%$ of tuning range from a $\pm 20 \%$-range core VCO [20]. The proposed architecture utilizes a differential VCO to generate the 


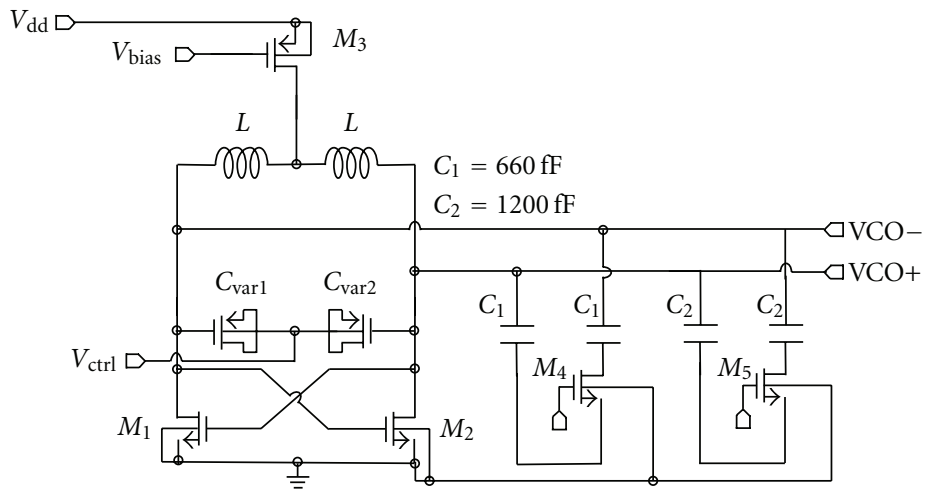

FIGURE 4: Schematics of core VCO using switched capacitors.

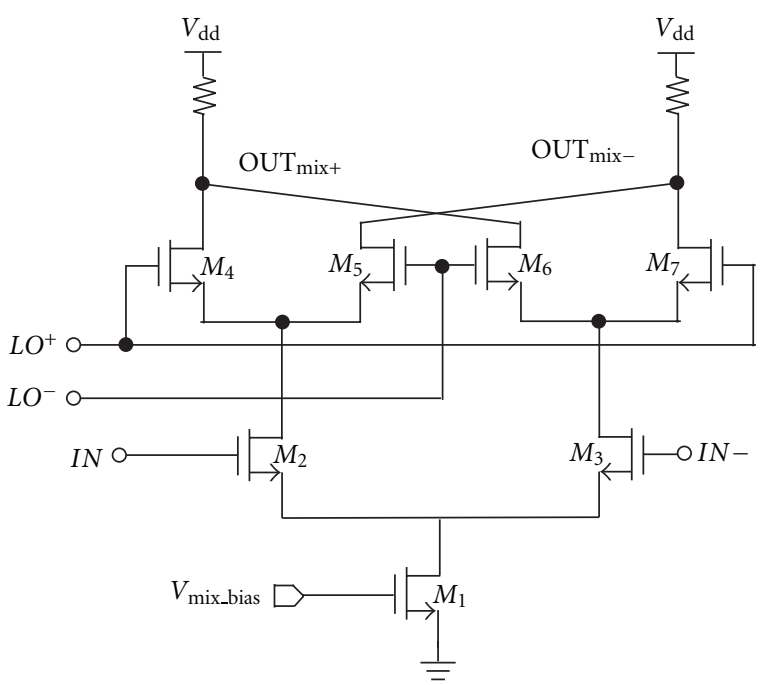

(a) wideband mixer

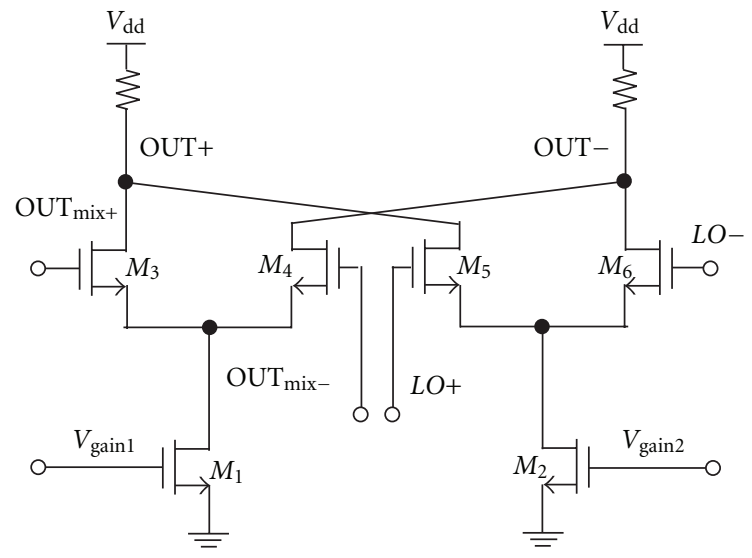

(b) variable gain combiner

Figure 5: Circuit schematics used in the proposed wideband VCO.

fundamental frequency with smaller layout area, lower power consumption, and lower phase noise characteristics than quadrature VCOs. A variable gain combiner is employed to reject spur instead of SSBM.

\section{Wideband VCO Architecture}

Figure 2 shows the proposed VCO architecture, which consists of a core VCO, two dividers, a switch, a mixer, a high-pass filter, and a combiner [20]. The proposed architecture aims to achieve wider tuning range with lower power and lower phase noise, so a differential VCO and a novel compact frequency extension circuit are introduced. Figure 3 shows frequency plan of the proposed architecture, and $2 f_{0}, 3 / 2 f_{0}, 3 / 4 f_{0}$, and $1 / 2 f_{0}$ are generated from the fundamental frequency $f_{0}$ of the core VCO. $2 f_{0}$ is generated by the mixer, and $1 / 2 f_{0}$ is divided from the fundamental frequency $f_{0} .3 / 2 f_{0}$ is generated from $f_{0}$ and $1 / 2 f_{0}$, and $1 / 2 f_{0}$ is also generated as a spurious signal. $3 / 4 f_{0}$ is divided from $3 / 2 f_{0}$. The core VCO is required to have frequency tuning range of $\pm 20 \%$, and the total tuning range of $\pm 71 \%$ can be realized by the frequency extension circuit. For example, tuning range of $2-3 \mathrm{GHz}$ can be extended to $1-6 \mathrm{GHz}$ as shown in Figure 3. Lower frequency can also be generated by a divide-by-2 frequency divider chain [3].

A differential VCO is employed as the core VCO. Figure 4 shows the schematic of the core VCO, and switched capacitors are utilized for coarse tuning. The differential VCO has better phase noise characteristic than the quadrature VCO, and smaller layout area and lower power consumption can also be achieved. The core VCO has frequency tuning range of more than $\pm 20 \%$. At higher frequencies, it is difficult to achieve wide tuning range due to parasitic capacitances, so the lower fundamental frequency is chosen and upconverted to higher frequencies by the mixer.

A CML divider is used as a wideband frequency divider to obtain 1/2 of input frequency, and a wideband mixer shown in Figure 5(a) is used as a frequency multiplier. The mixer is shared to generate $2 f_{0}$ and $3 / 2 f_{0}$, and input signal of mixer is switched as shown in Figure 2. In case (A) shown in Figure 2, mixer input signals are $f_{0}$ and $1 / 2 f_{0}$, and $3 / 2 f_{0}$ and $1 / 2 f_{0}$ are generated. In case (B) shown in Figure 2, both mixer input signals are $f_{0}$, and $\mathrm{DC}$ and $2 f_{0}$ are generated.

In case (A), $3 / 2 f_{0}$ is the desired frequency and $1 / 2 f_{0}$ is spurious frequency. The tuning range extension using SSBM requires $I / Q$ phases to reject the spurious frequency. In the proposed architecture, output of the first divider has 


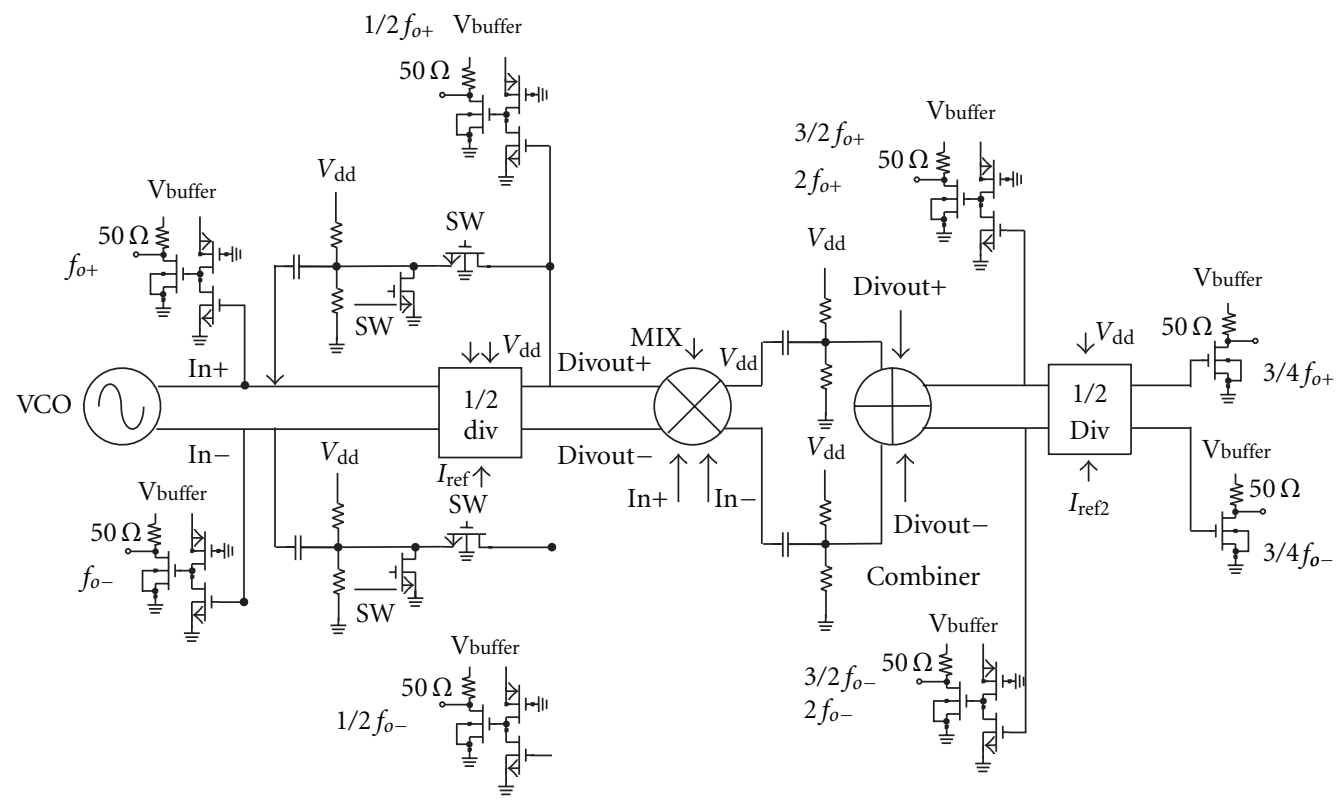

Figure 6: Block diagram of the proposed wideband VCO.

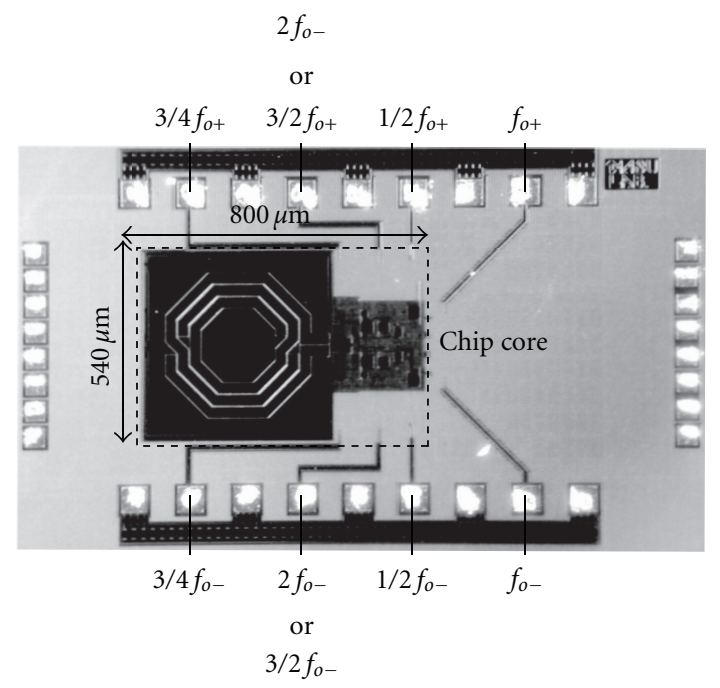

FIGURE 7: Chip micrograph of fabricated wideband VCO. Core size is $540 \mu \mathrm{m} \times 800 \mu \mathrm{m}$.

the same frequency as $1 / 2 f_{0}$ of spur, and it can be used for the spurious rejection instead of the SSBM technique. Therefore, the proposed architecture does not need QVCO and SSBM, and small-size synthesizer can be realized. First, the spurious frequency is rejected by the high-pass filter shown in Figure 2. Second, the remaining spur in the output of filter is rejected by a variable gain combiner shown in Figure 5(b). The gains of combiner are adjusted by bias voltages $V_{\text {gain } 1}$ and $V_{\text {gain2 } 2}$. The high-pass filter is also used for phase adjustment, and the filter should be carefully designed to reduce phase mismatch in wide frequency range.

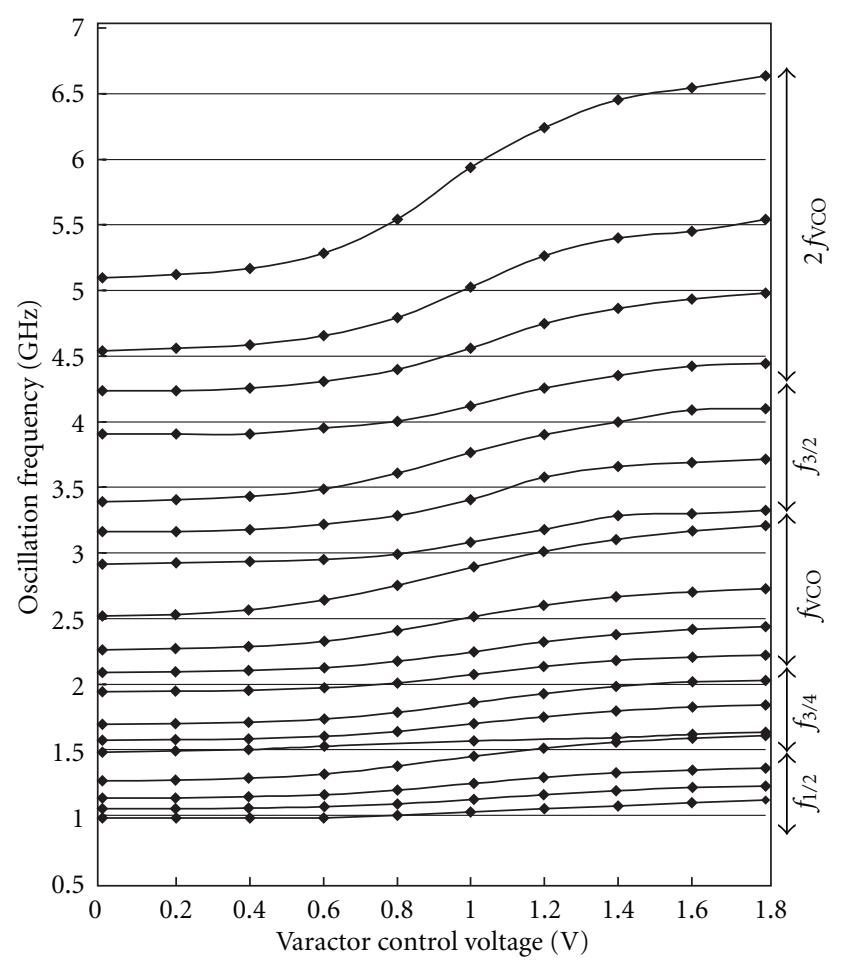

FIGURE 8: Measured tuning characteristics of the proposed VCO, which exhibits from $0.98 \mathrm{GHz}$ to $6.6 \mathrm{GHz}$ oscillation (149\%).

In case (B), $2 f_{0}$ is the desired frequency and DC signal is spurious. The DC signal can be suppressed by the high-pass filter. In the proposed architecture, distance to spur is large, which is a desirable feature for spurious rejection in both cases (A) and (B). The proposed architecture is also expected to be robust for LO leak. 


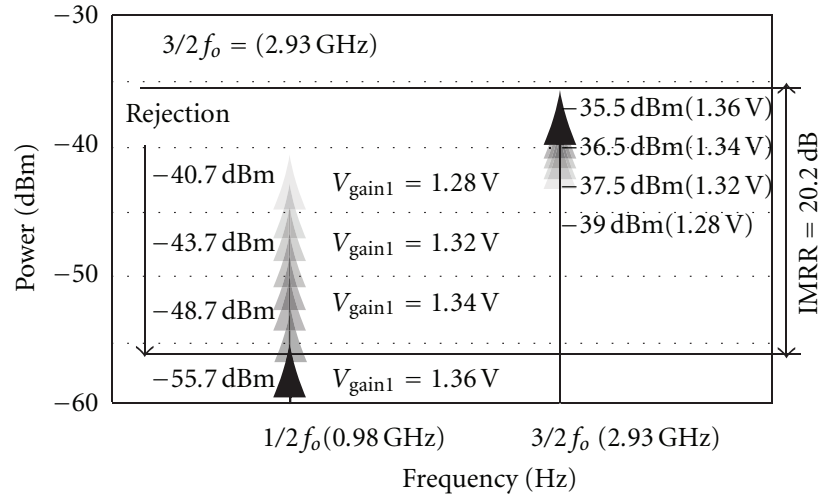

FIGURE 9: Spectrum of combiner output including $3 / 2 f_{0}$ and $1 / 2 f_{0}$ frequencies. The spurious rejection is performed by the high-pass filter and the variable gain combiner.

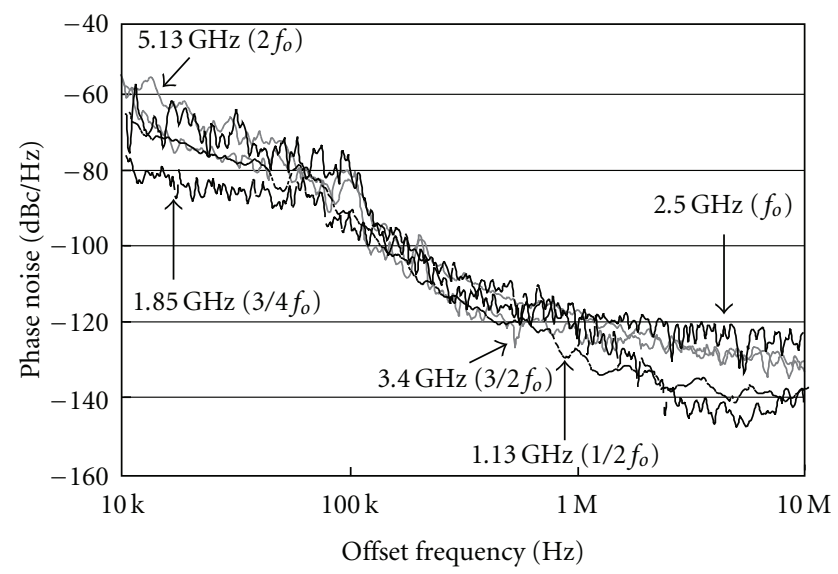

Figure 10: Phase noise at $f_{0}(2.50 \mathrm{GHz})$ and $3 / 4 f_{0}(1.85 \mathrm{GHz})$.

TABLE 1: VCO performance summary.

\begin{tabular}{ll}
\hline Technology & $\begin{array}{l}\text { TSMC } 0.18 \mu \mathrm{m} \text { CMOS process with RF } \\
\text { option }\end{array}$ \\
\hline Supply voltage $V_{\mathrm{DD}}$ & $1.8 \mathrm{~V}$ \\
VCO core current & $2.45 \sim 14.9 \mathrm{~mA}$ \\
Power consumption & $4.41 \sim 26.9 \mathrm{~mW}$ \\
Center frequency & $3.81 \mathrm{GHz}$ \\
Tuning range & $0.98 \mathrm{GHz} \sim 6.64 \mathrm{GHz} 149 \%$ \\
Chip area & $800 \mu \mathrm{m} \times 540 \mu \mathrm{m}$ \\
\hline
\end{tabular}

Figure 6 shows the detailed block diagram of the proposed wideband VCO. $2 f_{0}, 3 / 2 f_{0}, 3 / 4 f_{0}$, and $1 / 2 f_{0}$ are output from each node as shown in Figure 6. In the measurement, $I / O$ buffers are utilized for each output. For an actual use, a selector is required, and some switching time is required for the frequency selection.

\section{Measurement Result}

Figure 7 shows a chip micrograph of the proposed wideband VCO, which is fabricated by using a $0.18 \mu \mathrm{m}$ CMOS process.
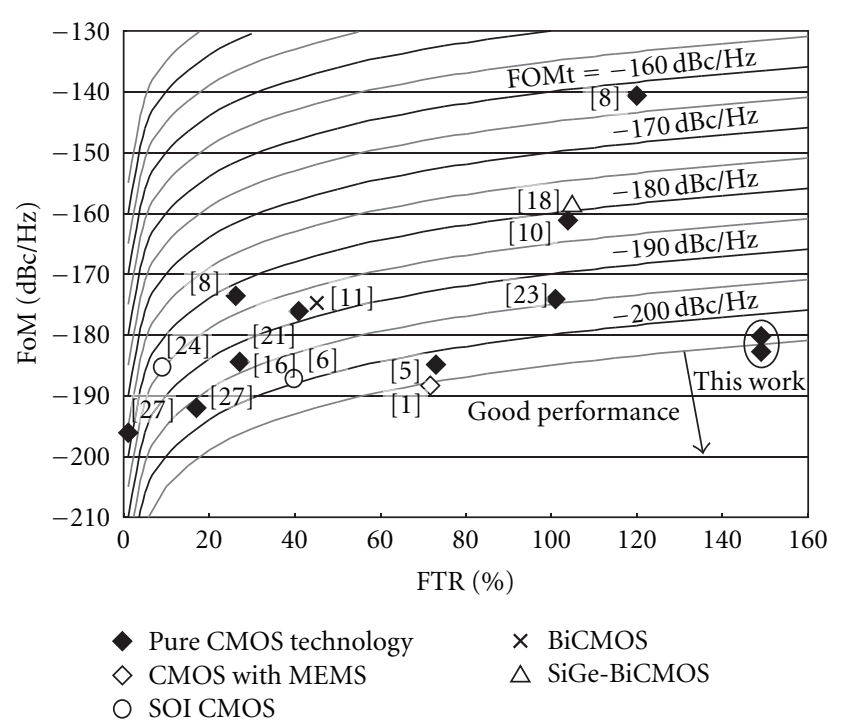

Figure 11: VCO performance comparison using FoM and frequency tuning range (FTR) [5-8, 10, 11, 16, 18, 21, 24-26].

TABLE 2: Phase noise performances.

\begin{tabular}{llll}
\hline $\begin{array}{l}\text { Oscillation } \\
\text { frequency }\end{array}$ & $\begin{array}{l}\text { Phase noise } \\
\text { @1 MHz offset }\end{array}$ & FoM & FoM \\
\hline $5.12 \mathrm{GHz}\left(2 f_{0}\right)$ & $-117 \mathrm{dBc} / \mathrm{Hz}$ & $-179 \mathrm{dBc} / \mathrm{Hz}$ & $-203 \mathrm{dBc} / \mathrm{Hz}$ \\
$3.40 \mathrm{GHz}\left(3 / 2 f_{0}\right)$ & $-122 \mathrm{dBc} / \mathrm{Hz}$ & $-179 \mathrm{dBc} / \mathrm{Hz}$ & $-203 \mathrm{dBc} / \mathrm{Hz}$ \\
$2.50 \mathrm{GHz}\left(f_{0}\right)$ & $-125 \mathrm{dBc} / \mathrm{Hz}$ & $-183 \mathrm{dBc} / \mathrm{Hz}$ & $-206 \mathrm{dBc} / \mathrm{Hz}$ \\
$1.85 \mathrm{GHz}\left(3 / 4 f_{0}\right)$ & $-128 \mathrm{dBc} / \mathrm{Hz}$ & $-180 \mathrm{dBc} / \mathrm{Hz}$ & $-203 \mathrm{dBc} / \mathrm{Hz}$ \\
$1.13 \mathrm{GHz}\left(1 / 2 f_{0}\right)$ & $-130 \mathrm{dBc} / \mathrm{Hz}$ & $-179 \mathrm{dBc} / \mathrm{Hz}$ & $-202 \mathrm{dBc} / \mathrm{Hz}$ \\
\hline
\end{tabular}

Core size is $800 \mu \mathrm{m} \times 540 \mu \mathrm{m}$. Depicted in Figure 7, the core area is dominated by the spiral inductor for LC-VCO. Signal Source Analyzer (Agilent E5052A) and Spectrum Analyzer (Agilent 8563EC) were used for measurement. GSG probes were also used to obtain on-chip signals. Figure 8 shows the tuning characteristics of the VCO, which exhibits $0.98 \mathrm{GHz}$ to $6.6 \mathrm{GHz}$ oscillation. The right $y$ axis shows the frequency coverage of each output path. The tuning range is found to be $149 \%$. Table 1 summarizes the measured results.

Figure 9 shows spectrum of the combiner output, which contains $3 / 2 f_{0}$ and $1 / 2 f_{0}$ of frequency. In this case, $3 / 2 f_{0}$ is $2.93 \mathrm{GHz}$. The spurious frequency of $1 / 2 f_{0}$ is rejected by both the high-pass filter and the variable gain combiner. The total image rejection ratio (IMRR) is $20.2 \mathrm{~dB}$. In this measurement, the bias voltages in the variable gain combiner were manually adjusted.

Figure 10 shows measured phase noise characteristics for $f_{0}$ as the fundamental frequency and $3 / 4 f_{0}$ as the final output. The $3 / 4 f_{0}$ signal is generated through all the circuit blocks shown in Figure 2. This result shows that the proposed wideband VCO operates with the wideband and the low phase noise. Table 2 summarizes the measured phase noise and FoM. The proposed VCO achieves $-183 \mathrm{dBc} / \mathrm{Hz}$ of FoM for $2.50 \mathrm{GHz}$ oscillation. In this paper, $\mathrm{FoM}_{\mathrm{T}}$ is also 
employed to evaluate tuning range in addition to the phase noise. $\mathrm{FoM}_{\mathrm{T}}$ is defined as the following equation [22]:

$$
\begin{aligned}
\text { FoM }_{\mathrm{T}}= & \text { FoM }-20 \log \left(\frac{\mathrm{FTR}}{10}\right) \\
= & \mathcal{L}\left\{f_{\text {offset }}\right\}-20 \log \left\{\frac{f_{0}}{f_{\text {offset }}} \cdot \frac{\text { FTR }}{10}\right\} \\
& +10 \log \left(\frac{P_{\mathrm{DC}}}{1 \mathrm{~m} W}\right),
\end{aligned}
$$

where $\mathcal{L}\left\{f_{\text {offset }}\right\}$ is phase noise, $f_{\text {offset }}$ is certain frequency offset, $f_{o}$ is center frequency, and $P_{\mathrm{DC}}$ is power consumption. FTR is frequency tuning range, which is defined as $\left(f_{\max }-\right.$ $\left.f_{\min }\right) / f_{0}$. Table 2 also shows $\mathrm{FoM}_{\mathrm{T}}$, and the proposed VCO achieves $-206 \mathrm{dBc} / \mathrm{Hz}$ of $\mathrm{FoM}_{\mathrm{T}}$ for $2.50 \mathrm{GHz}$ oscillation.

Figure 11 plots performances of wideband LC-VCO reported in the literature $[5-8,10,11,16,18,21,24-$ 26], which includes low phase noise VCOs using SOI [24, 25] and BiCMOS processes [6] and CMOS VCOs using phase noise improvement techniques $[5,11]$. The proposed VCO achieves the widest tuning range and the best $\mathrm{FoM}_{\mathrm{T}}$ simultaneously.

\section{Conclusion}

This paper has proposed a novel wideband LC-VCO for multiband applications. The VCO has the core VCO and the tuning range extension circuit. A differential LC-VCO and a double-balanced mixer are utilized instead of a quadrature VCO and a single-sideband mixer for the spurious rejection. In measurement results, the proposed VCO performs 0.98 to $6.6 \mathrm{GHz}$ continuous frequency tuning with $-206 \mathrm{dBc} / \mathrm{Hz}$ of $\mathrm{FoM}_{\mathrm{T}}$, which is fabricated by using a $0.18 \mu \mathrm{m}$ CMOS process. The frequency tuning range (FTR) is $149 \%$, and the chip area is $800 \mu \mathrm{m} \times 540 \mu \mathrm{m}$. The proposed VCO achieves the widest tuning range and the best $\mathrm{FoM}_{\mathrm{T}}$.

\section{Acknowledgments}

This work was partially supported by JSPS.KAKENHI, STARC, MIC.SCOPE, and VDEC in collaboration with Cadence Design Systems, Inc.

\section{References}

[1] D. Leenaerts, R. van de Beek, G. van der Weide et al., "A SiGe BiCMOS 1ns fast hopping frequency synthesizer for UWB radio," in Proceedings of the IEEE International Solid-State Circuits Conference, (ISSCC '05), pp. 202-593, February 2005.

[2] J. Lee, "A 3-to-8 GHz fast-hopping frequency synthesizer in $0.18 \mathrm{x} \mu \mathrm{m}$ CMOS technology," IEEE Journal of Solid-State Circuits, vol. 41, no. 3, pp. 566-573, 2006.

[3] Y. Kobayashi, K. Ohashi, Y. Ito, H. Ito, K. Okada, and K. Masu, "A $0.49-6.50 \mathrm{GHz}$ wideband LC-VCO with high-IRR in a $180 \mathrm{~nm}$ CMOS technology," in Proceedings of the International Conference on Solid State Devices and Materials, pp. 268-269, September 2007.

[4] B. Razavi, T. Aytur, C. Lam et al., "Multiband UWB transceivers," in Proceedings of the IEEE Custom Integrated Circuits Conference, pp. 140-147, September 2005.
[5] C. Yao and A. Willson, "A phase-noise reduction technique for quadrature LC-VCO with phase-to-amplitude noise conversion," in Proceedings of the IEEE International Solid-State Circuits Conference, (ISSCC '06), pp. 196-191, February 2006.

[6] P. Vaananen, M. Metsanvirta, and N. T. Tchamov, " $4.3 \mathrm{GHz}$ VCO with $2 \mathrm{GHz}$ tuning range and low phase noise," IEEE Journal of Solid-State Circuits, vol. 36, no. 1, pp. 142-146, 2001.

[7] A. Fard, T. Johnson, and D. Aberg, "A low power wide band CMOS VCO for multi-standard radios," in Proceedings of IEEE Radio and Wireless Conference, pp. 79-82, September 2004.

[8] R. Mukhopadhyay, Y. Park, P. Sen et al., "Reconfigurable RFICs in Si-based technologies for a compact intelligent RF frontend," IEEE Transactions on Microwave Theory and Techniques, vol. 53, no. 1, pp. 81-91, 2005.

[9] H. Tsurumi and Y. Suzuki, "Broadband RF stage architecture for software-defined radio in handheld terminal applications," IEEE Communications Magazine, vol. 37, no. 2, pp. 90-95, 1999.

[10] D. Guermandi, P. Tortori, E. Franchi, and A. Gnudi, "A 0.83$2.5 \mathrm{GHz}$ continuously tunable quadrature VCO," IEEE Journal of Solid-State Circuits, vol. 40, no. 12, pp. 2620-2627, 2005.

[11] A. D. Berny, A. M. Niknejad, and R. G. Meyer, "A $1.8 \mathrm{GHz}$ LC VCO with $1.3 \mathrm{GHz}$ tuning range and digital amplitude calibration," IEEE Journal of Solid-State Circuits, vol. 40, no. 4, pp. 909-916, 2005.

[12] H. Eul, "ICs for mobile multimedia communications," in Proceedings of the IEEE International Solid-State Circuits Conference, (ISSCC '06), pp. 31-20, February 2006.

[13] R. Bagheri, A. Mirzaei, S. Chehrazi et al., "An $800 \mathrm{MHz}$ to $5 \mathrm{GHz}$ software-defined radio receiver in $90 \mathrm{~nm}$ CMOS," in Proceedings of the IEEE International Solid-State Circuits Conference, (ISSCC '06), pp. 469-480, February 2006.

[14] Y. Yoshihara, H. Sugawara, H. Ito, K. Okada, and K. Masu, "Reconfigurable RF circuit design for multi-band wireless chip," in Proceedings of 2004 IEEE Asia-Pacific Conference on Advanced System Integrated Circuits, pp. 418-419, jpn, August 2004.

[15] K. Okada, Y. Yoshihara, H. Sugawara, and K. Masu, "A dynamic reconfigurable RF circuit architecture," in Proceedings of the IEEE/ACM Asia and South Pacific Design Automation Conference, pp. 683-686, 2005.

[16] A. Kral, F. Behbahani, and A. A. Abidi, "RF-CMOS oscillators with switched tuning," in Proceedings of the IEEE Custom Integrated Circuits Conference, pp. 555-557, May 1998.

[17] C. Liang, S. Liu, Y. Chen, T. Yang, and G. Ma, "A 14-band frequency synthesizer for MB-OFDM UWB application," in Proceedings of the IEEE International Solid-State Circuits Conference, (ISSCC '06), pp. 113-126, February 2006.

[18] Y. Ito, Y. Yoshihara, H. Sugawara, K. Okada, and K. Masu, "A $1.3-2.8 \mathrm{GHz}$ wide range CMOS LC-VCO using variable inductor," in Proceedings of the IEEE Asian Solid-State Circuits Conference Digest of Technical Papers, pp. 265-268, November 2005.

[19] B. Razavi, "Multi-decade carrier generation for cognitive radios," in Proceedings of the Symposium on VLSI Circuits, pp. 120-121, June 2009.

[20] Y. Ito, H. Sugawara, K. Okada, and K. Masu, "A 0.98 to $6.6 \mathrm{GHz}$ tunable wideband VCO in a $180 \mathrm{~nm}$ CMOS technology for reconfigurable radio transceiver," in Proceedings of the IEEE Asian Solid-State Circuits Conference Digest of Technical Papers, pp. 359-362, November 2006.

[21] A. D. Berny, A. M. Niknejad, and R. G. Meyer, "A wideband low-phase-noise CMOS VCO," in Proceedings of the IEEE 
Custom Integrated Circuits Conference, pp. 555-558, September 2003.

[22] A. Ismail and A. Abidi, "A 3.1- To 8.2 GHz zero-IF receiver and direct frequency synthesizer in $0.18 \mu \mathrm{m}$ SiGe BiCMOS for mode-2 MB-OFDM UWB communication," IEEE Journal of Solid-State Circuits, vol. 40, no. 12, pp. 2573-2582, 2005.

[23] P. Nuzzo, K. Vengattaramane, M. Ingels, V. Giannini, M. Steyaert, and J. Craninckx, "A 0.1-5 GHz dual-VCO softwaredefined $\Sigma \Delta$ frequency synthesizer in $45 \mathrm{~nm}$ digital CMOS," in Proceedings of the IEEE Radio Frequency Integrated Circuits Symposium, (RFIC '09), pp. 321-324, June 2009.

[24] N. Fong, J. Plouchart, N. Zamdmer et al., "A 1V 3.8-5.7 GHz differentially-tuned VCO in SOI CMOS," in Procedings of the IEEE Radio Frequency Integrated Circuits Symposium, Digest of Papers, pp. 75-78, June 2002.

[25] J. Kim, J. O. Plouchart, N. Zamdmer et al., "A $44 \mathrm{GHz}$ differentially tuned VCO with $4 \mathrm{GHz}$ tuning range in $0.12 \mu \mathrm{m}$ SOI CMOS," in Proceedings of the IEEE International SolidState Circuits Conference, (ISSCC '05), pp. 416-607, February 2005.

[26] A. Tanaka, H. Okada, H. Kodama, and H. Ishikawa, "A $1.1 \mathrm{~V}$ 3.1-to-9.5 GHz MB-OFDM UWB transceiver in $90 \mathrm{~nm}$ CMOS," in Proceedings of the IEEE International Solid-State Circuits Conference, (ISSCC '06), pp. 120-113, February 2006. 

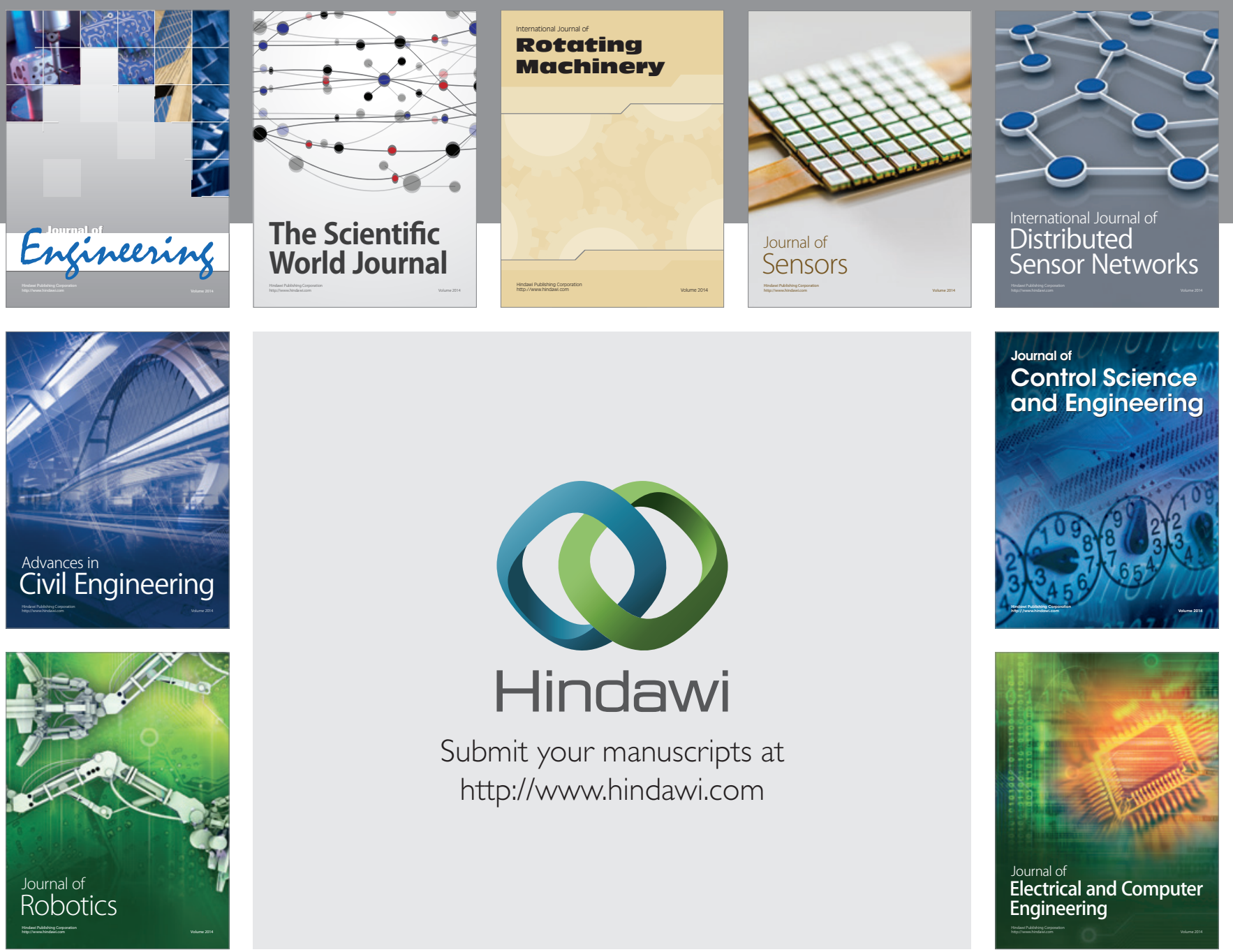

Submit your manuscripts at

http://www.hindawi.com
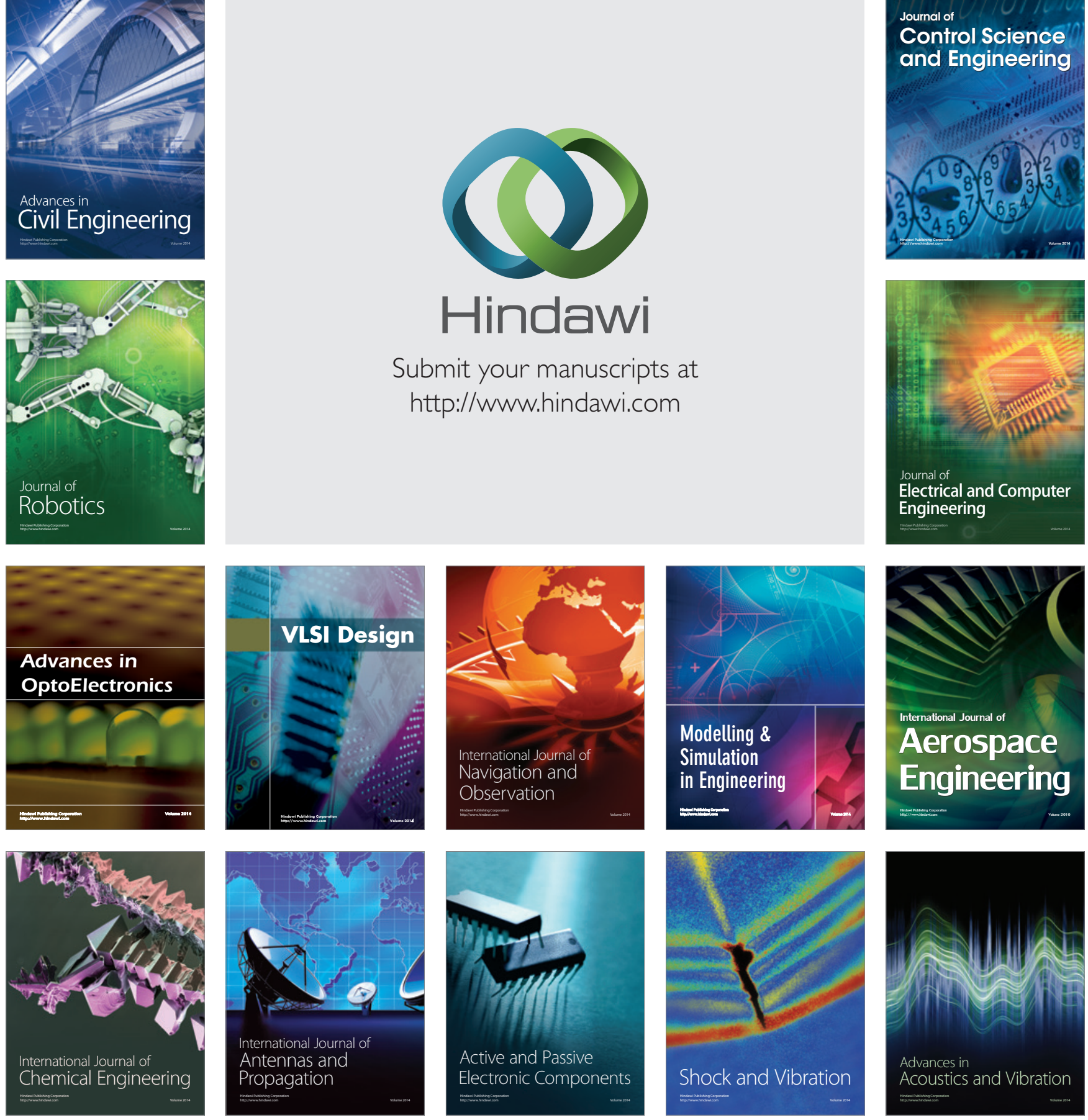